\title{
Gene Expression of Antioxidant Enzymes in the Resected Intestine in Crohn's Disease
}

\author{
Otakar Sotona ${ }^{1,2, *}$, Eva Peterová3 ${ }^{3}$ Július Örhalmi², Tomáš Dušek¹,2, Alena Mrkvicová3, \\ Veronika Knoblochová4, Petr Lochman ${ }^{1,2}$, Ondřej Malý1,2, Jiř́ Páral ${ }^{1,2}$, Jan Bureš ${ }^{4}$
}

\begin{abstract}
Introduction: The inflammatory process in Crohn's disease (CD) is closely associated with the formation of reactive oxygen species. Antioxidant enzymes can play an important role in the outcome of CD and may influence postoperative recurrence in these patients. The aim of our study was to evaluate gene expression of intracellular antioxidant enzymes in surgically resected intestinal specimens of patients with $C D$, both in macroscopically normal and in inflamed tissue.

Methods: A total of 28 patients referred for elective bowel resection were enrolled in the study. Full-thickness small intestinal specimens were investigated. Gene expression of antioxidant enzymes - superoxide dismutase (SOD), glutathione peroxidase (GPX), glutathione reductase (GSR) - was evaluated both in macroscopically normal and inflamed samples.

Results: There were significantly lower levels of SOD1 mRNA ( $p=0.007)$ and GSR mRNA ( $p=0.027)$ in inflamed tissue compared to macroscopically normal areas. No significant differences were found between affected and non-affected intestinal segments in mRNA for SOD2, SOD3 and GPX.

Conclusions: Our pilot data clearly showed that the gene expression of major antioxidant enzymes is not a uniform mechanism in the pathogenesis of Crohn's disease. Topically decreased gene expression of SOD1 and GSR might facilitate the segmental tissue injury caused by reactive oxygen species.

\section{KEYWORDS}

Crohn's disease; gene expression; intestine; antioxidant enzymes; superoxide dismutase; glutathione peroxidase; glutathione reductase

AUTHOR AFFILIATIONS

${ }^{1}$ Department of Field Surgery, Faculty of Military Health Sciences, University of Defence, Hradec Králové, Czech Republic

2 Department of Surgery, Charles University, Faculty of Medicine in Hradec Králové and University Hospital Hradec Králové, Czech Republic

${ }^{3}$ Department of Medical Biochemistry, Charles University, Faculty of Medicine in Hradec Králové, Czech Republic

${ }^{4}$ 2nd Department of Internal Medicine - Gastroenterology, Charles University, Faculty of Medicine in Hradec Králové and University Hospital Hradec Králové, Czech Republic

* Corresponding author: Department of Field Surgery, Faculty of Military Health Sciences, University of Defence, Hradec Králové, Czech Republic; e-mail: otakar.sotona@seznam.cz
\end{abstract}




\section{INTRODUCTION}

Inflammatory bowel disease (IBD) is comprised of two entities: ulcerative colitis and Crohn's disease (CD). Ulcerative colitis affects the large bowel only, whereas $C D$ can involve any part of the gastrointestinal tract (most commonly the ileum and proximal colon). These diseases have somewhat different pathologic and clinical characteristics, but with substantial overlap. $C D$ is characterized by transmural inflammation and by segmental involvement of the bowel. The transmural inflammation may lead to fibrosis and strictures, and to obstructive clinical presentations, and may also result in sinus tracts, giving rise to microperforations and fistula formation $(1,2)$.

The pathogenesis of IBD still remains poorly understood. Several studies both in humans and in animal models suggest that genetically determined factors contribute to susceptibility to CD. However, only about 15 percent of those with $C D$ have family history of IBD. The pathogenesis of $C D$ results from dysregulated immune responses to luminal bacteria (and/or their products) and from the impact of various environmental factors (e.g., dietary factors, xenobiotics, smoking, drugs, oral contraceptives in women, and others). The components of immune response must be properly balanced. Either too strong or inadequate immune response to microbes in the intestinal lumen can ultimately result in intestinal inflammation (3).

Immune response in $\mathrm{CD}$ is a complex process, comprising dysregulated all innate-immune-, cytokine-, adaptive-immune-, epithelial-barrier- and microbial-clearance pathways. Inflammatory reaction, among others mediated by polymorphonuclear neutrophils, is associated with production of several products that can cause endothelial dysfunction and further worsen the structural intestinal injury. Reactive oxygen species (ROS) are key signalling molecules that play an important role in this process. Oxidative stress produced by polymorphonuclears leads to the opening of inter-endothelial junctions and promotes the migration of inflammatory cells across the endothelial barrier (4).

Antioxidant enzymes are proteins involved in the catalytic transformation of free radicals and reactive oxygen species and their by-products into stable nontoxic molecules. They represent an important defence mechanism against oxidative stress-induced cell damage. These enzyme systems include superoxide dismutase, glutathione peroxidase, glutathione reductase, lipoic acids, peroxiredoxins and catalases (5).

Although advances in medical therapy have been associated with decreased need for bowel resection in $C D$, surgical intervention is often required in the setting of bowel obstruction, abscesses or fistulas, or refractory disease. The 10-year risk of surgical resection for $\mathrm{CD}$ is nearly 50 percent (6).

Antioxidant enzymes may play an important role in the outcome of $C D$ and may influence the postoperative recurrence in these patients. The aim of our current study was to evaluate gene expression of intracellular antioxidant enzymes in surgically resected intestinal specimens of patients with CD, both in macroscopically uninvolved and involved tissue.

\section{METHODS}

\section{PATIENTS}

A total of 28 individuals referred for bowel resection were included in the study. The group consisted of 12 males (mean age $37 \pm 13 ; 6 / 12$ were smokers) and 16 females (mean age $38 \pm 13 ; 8 / 16$ smokers).

\section{SURGICAL PROCEDURE}

All patients were indicated for intestinal resection according to the decision of a multidisciplinary team. All patients had L3B2 disease according to the Montreal classification (7). No preoperative bowel preparation was performed. A prophylactic dose of antibiotics was administered (amoxicillin/clavulanate $1.2 \mathrm{~g}$ i.v., metronidazole $500 \mathrm{mg}$ i.v.). Patients underwent laparoscopic or open ileocecal resection or right hemicolectomy with a hand-sutured side-to-side or end-to-end anastomosis. For each patient, a full-thickness sample from small intestine was taken from the resected specimen by a surgeon from a macroscopically uninvolved and affected area. Samples were immediately stored in separate vials with RNAprotect Tissue Reagent (QIAGEN, Hilde, Germany) and were ready for further processing.

\section{ANTIOXIDANT ENZYMES}

Analysis of mRNA expression of major intracellular antioxidant enzymes was performed, both in macroscopically normal and in involved tissue. Analyses were carried out of superoxide dismutase (SOD) and its three isoforms - cytoplasmatic (SOD1), mitochondrial (SOD2) and extracellular (SOD3). Gene expression of glutathione peroxidase (GPX) and glutathione reductase (GSR) was also investigated.

\section{RNA ISOLATION AND QPCR}

Tissue samples were homogenized using a Precellys 24 homogenizer (Bertin Instruments, Bretonneux, France). Total cellular RNA was extracted using TRIzol reagent (Invitrogen, Carlsbad, CA, USA). RNA was reverse-transcribed using a cDNA Reverse Transcription Kit (Applied Biosystems, Foster City, CA, USA). Gene expression was quantified with TaqMan Gene Expression Assays (POLR2A Hs00172187_m1, SOD1 HS_00916176_m1, SOD2 Hs_00167309_m1, SOD3 Hs_00162090_m1, GSR HS_00167317_m1, GPX3 HS_01078668_m1. Gene expression was analysed using a QuantStudio 6 real-time PCR system (all purchased from Applied Biosystems, Foster City, CA, USA). Results were normalized to POLR2A RNA expression. mRNA levels were calculated using a comparative $\mathrm{Ct}$ method ( $\Delta \Delta \mathrm{Ct}$ method) (8).

\section{STATISTICAL ANALYSIS}

Data had non-normal distribution and were tested by Mann-Whitney test and Kruskal-Wallis test. All statistics was performed using GraphPad Prism 8.0.1.244 (San Diego, CA, USA). 


\section{ETHICS}

The project was carried out according to the Declaration of Helsinki and was approved by the Ethics Committee of University Hospital Hradec Králové (protocol number 201706S12P). All participants signed an informed consent.

\section{RESULTS}

There was a statistically significant difference between macroscopically non-pathological and pathological tissue in SOD1 mRNA level ( $p=0.007$; Figure 1.A) and GSR mRNA level ( $p=0.026$; Figure 1.D). No significant differences were found between macroscopically involved and non-involved intestinal samples in mRNA for SOD2 (Figure 1.B), SOD3 ( $\mathrm{p}=0.116$; type 2 error beta: 0.783; power of the performed test 0.217; Figure 1.C), or GPX (Figure 1.E).

The mRNA for each enzyme in the non-pathological and pathological tissue of the males group was compared to the mRNA for each enzyme in females group. No statistically significant differences in the mRNA levels were found between male and female non-pathological tissue for SOD1 $(p=0.932)$, SOD2 $(p=0.819)$, SOD3 $(p=0.357)$, GSR $(p=0.948)$, or GPX ( $p=0.765)$. No statistical differences were found between male non-pathological and pathological tissue in mRNA for SOD1 ( $p=0.981)$, SOD2 $(p=0.999)$, SOD3 $(p=0.995)$, GSR $(p=0.893)$, or GPX $(p=0.769)$, nor were any differences identified between female non-pathological and pathological tissue in mRNA for SOD1 $(p=0.918)$, SOD2 $(p=0.593)$, SOD3 $(p=0.235)$, GSR ( $p=0.525)$, or GPX $(p=0.340)$.

The mRNA for each enzyme in the non-pathological and pathological tissue of the smokers was compared to the mRNA for each enzyme in non-smokers. No statistically significant differences in the mRNA levels were found in non-pathological tissue for SOD1 $(p=0.950)$, SOD2 $(p=$ $0.962)$, SOD3 ( $p=0.613)$, GSR $(p=0.472)$, GPX $(p=0.738)$ between smokers and non-smokers. For non-pathological and pathological tissue in smokers, no statistical differences in mRNA levels for SOD1 ( $p=0.999)$, SOD2 $(p=$ $0.944), \operatorname{SOD} 3(p=0.240), \operatorname{GSR}(p=0.995), \operatorname{GPX}(p=0.993)$ were identified, nor were there any differences between non-smokers non-pathological and pathological tissue in mRNA for SOD1 $(\mathrm{p}=0.783)$, SOD2 $(\mathrm{p}=0.893)$, SOD3 $(\mathrm{p}=$ $0.985)$, GSR $(p=0.243), \operatorname{GPX}(p=0.925)$.

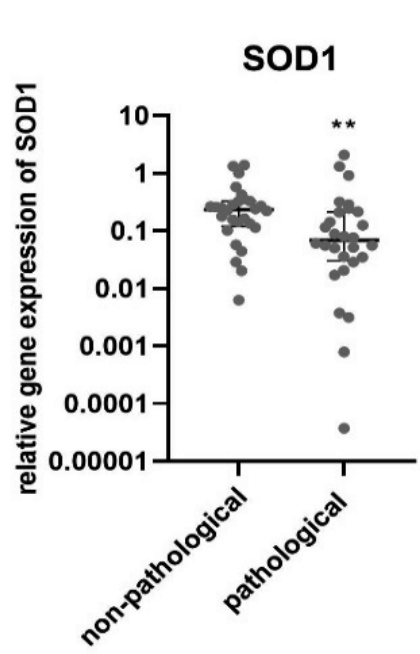

A

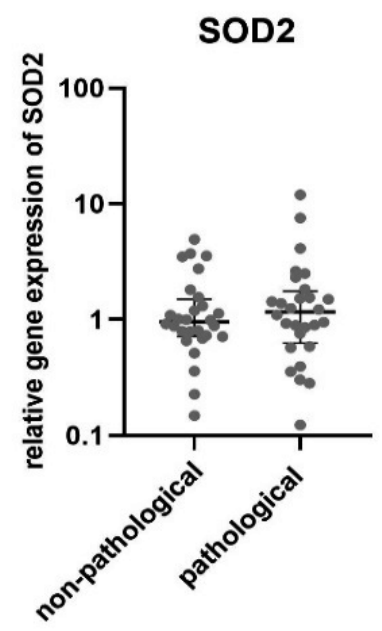

D
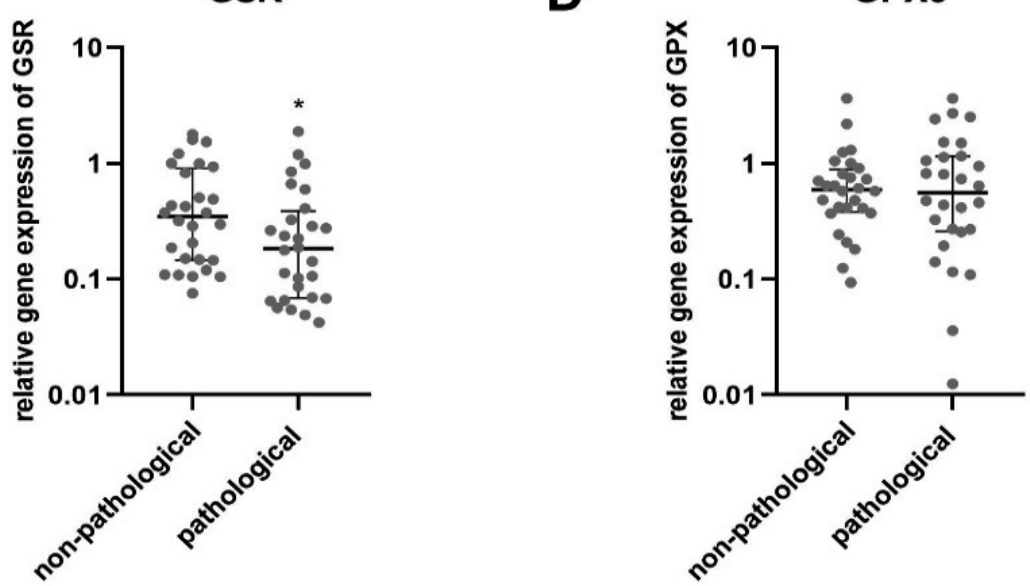

B

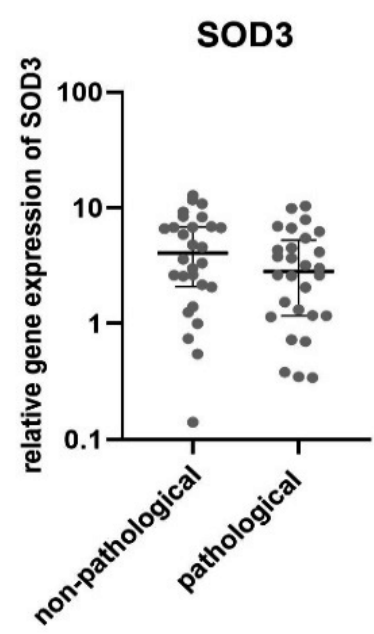

$\mathbf{E}$

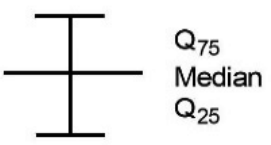

Fig. 1 mRNA expression of SOD1 (A), SOD2 (B), SOD3 (C), GSR (D), GPX (E) in macroscopically non-pathological tissue $(n=28)$ and macroscopically pathological tissue $(n=28)$. 


\section{DISCUSSION}

Our study brought important insights into the role of gene expression of antioxidant enzymes in Crohn's disease (CD). From the analysis of pathologically altered and macroscopically uninvolved tissue samples, a statistically significant difference in mRNA level of SOD1 was found. A significantly reduced level of SOD1 was noted in the affected intestinal samples. Superoxide dismutase catalyses the disproportionation of superoxide radicals into $\mathrm{H}_{2} \mathrm{O}_{2}$ and $\mathrm{O}_{2}$. SOD1 binds copper and zinc ions and is one of three superoxide dismutases. SOD1 deficiency in the dextran-sodium sulphate (DSS)-induced mouse model of colitis resulted in severe oxidative stress with body weight loss, epithelial barrier disruption, and decreased antioxidant enzyme activities (9). In CD, lipid peroxidation was found to correlate positively with SOD1 (10). The remaining two isoforms of SOD in our study failed to show a statistically significant difference in mRNA levels. However, Kruidenier et al. (11) demonstrated increased SOD2 expression in their work. SOD2 transforms toxic superoxide to clear mitochondrial reactive oxygen species, thus protecting against cell death. Mulder et al. (12) measured the content of superoxide dismutase in intestinal resection specimens from patients with Crohn's disease and ulcerative colitis and compared the concentrations with those obtained in the normal mucosa of a control group of patients with colorectal cancer. The superoxide dismutase content was similar in control mucosa and non-inflamed mucosa from that in patients with inflammatory bowel disease but was decreased in inflamed mucosa. The decreased SOD3 protein level in inflamed tissue compared to non-inflamed has been described in other studies $(11,13)$.

Our study also demonstrated a statistically significant decrease in glutathione reductase (GSR) mRNA levels. This enzyme regenerates the glutathione (GSH). GSR plays a key role in protecting cells from oxidative damage, it catalyses the reduction of $\mathrm{H}_{2} \mathrm{O}_{2}$ by GSH into $\mathrm{H}_{2} \mathrm{O}$ and glutathione disulphide (GSSG). The inflamed ileum of patients with $C D$ is characterized both by an increase of GSSG and decrease of GSH (14). The inflamed ileum in CD is not able to eliminate GSSG, probably due to a diminished GSR activity. Our results showed that such decrease is already evident at the gene level.

Glutathione peroxidase (GPX) was another enzyme whose gene expression was analysed in our study. GPX is involved in the protection of cells from damaging effects of reactive forms of oxygen. It follows the action of SOD. It catalyses the decomposition of $\mathrm{H}_{2} \mathrm{O}_{2}$ into water and oxygen (15). Our study did not confirm differences between inflamed and non-inflamed tissue in gene expression of GPX.

We have also investigated the possible influence of gender and smoking on the gene expression of antioxidant enzymes. Sex hormones, e.g. oestrogen, are thought to be associated with risk of IBD as variations in disease severity occur during pregnancy, menopause, or oral contraceptive use $(16,17)$. Unfer et al. (18) showed that serum oestrogen and progesterone levels positively correlated with blood SOD1 and SOD3 activity. Strehlow et al. (19) revealed that expression as well as activity of SOD2 and SOD3 are enhanced by oestrogens by transcriptional pathways, while
GPX is not altered. In our study, we found no differences between the genders in gene expression of antioxidant enzymes.

Cigarette smoking in $\mathrm{CD}$ is put into context with accelerated disease, worse nutritional status, increased need for medical therapy (including biologics), and increased risk of recurrence following surgery, as well as a higher risk of postoperative complications (20-22). Contrarily, smoking has a protective effect for ulcerative colitis, and smokers are less likely to require colectomy $(23,24)$. However, our results showed no differences between smokers and non-smokers in CD, both in macroscopically normal and pathological tissue.

There is an increasing interest in miRNAs and exploring their possible role in the pathogenesis of $\mathrm{CD}$. Current studies indicate that miRNA expression can be sensitive to the presence of intracellular $\mathrm{H}_{2} \mathrm{O}_{2}$ levels (25-27). Epigenetic regulation at the DNA level is an important mechanism involved in $\mathrm{H}_{2} \mathrm{O}_{2}$-mediated expression changes of multiple genes, indicating that miRNA expression is very sensitive to $\mathrm{H}_{2} \mathrm{O}_{2}$ stimulation. For example, in smooth muscle cells, cellular treatment with hydrogen peroxide resulted in an upregulation of microRNA-21 (10).

We are aware of possible limits of our study. We did not correlate our results with the histology of resected specimens (and tissue inflammatory grading). Due to the limited number of patients, we were not able to assess our data with respect to preoperative medical therapy. And last but not least, we did not evaluate other factors that can influence the gene expression of antioxidant enzymes and thus might create confounders. Nevertheless, our pilot data is an important basis for future research of this important topic.

\section{CONCLUSIONS}

Our pilot data clearly showed that the gene expression of major antioxidant enzymes is not a uniform mechanism in the pathogenesis of Crohn's disease. Topically decreased gene expression of SOD1 and GSR might facilitate the segmental tissue injury caused by reactive oxygen species.

\section{ACKNOWLEDGEMENTS}

This work was supported by the project PROGRES Q40-15 and Q40-01 from Charles University and by MH CZ - DRO (UHHK, 00179906).

The study was supported by A long-term organization development plan 1011 - Clinical fields (Faculty of Military Health Sciences 2016-2020).

The authors are grateful to Ian McColl, MD, $\mathrm{PhD}$ for assistance with the manuscript.

\section{REFERENCES}

1. Peppercorn MA, Cheifetz AS. Definitions, epidemiology, and risk factors for inflammatory bowel disease in adults. UpToDate on-line (Topic 4066, Version 38.0), Alphen aan den Rijn, Wolters Kluwer; accessed on 10th April 2021.

2. Lukáš M, et al. Idiopatické střevní záněty. Nové trendy a mezioborové souvislosti. Praha: Grada, 2020. 
3. Snapper SB, Abraham C. Immune and microbial mechanisms in the pathogenesis of inflammatory bowel disease. UpToDate on-line (Topic 4077, Version 22.0), Alphen aan den Rijn, Wolters Kluwer; accessed on 10th April 2021.

4. Mittal M, Siddiqui MR, Tran K, Reddy SP, Malik AB. Reactive oxygen species in inflammation and tissue injury. Antioxid Redox Signal 2014; 20(7): 1126-67.

5. Sáez GT, Están-Capell N. Antioxidant Enzymes. In: Schwab M. (eds) Encyclopedia of Cancer. Berlin: Springer, 2014.

6. Frolkis AD, Dykeman J, Negrón ME, et al. Risk of surgery for inflammatory bowel diseases has decreased over time: a systematic review and meta-analysis of population-based studies. Gastroenterology 2013; 145(5): 996-1006.

7. Silverberg MS, Satsangi J, Ahmad T, et al. Toward an integrated clinical, molecular and serological classification of inflammatory bowel disease: report of a Working Party of the 2005 Montreal World Congress of Gastroenterology. Can J Gastroenterol 2005; 19, Suppl A: $5 \mathrm{~A}-36 \mathrm{~A}$.

8. Rao X, Huang X, Zhou Z, Lin X. An improvement of the $2^{\wedge}$ (-delta delta CT) method for quantitative real-time polymerase chain reaction data analysis. Biostat Bioinform Biomath 2013; 3: 71-85.

9. Hwang J, Jing J, Sejin J, Shin HM, et al. SOD1 suppresses pro-inflammatory immune responses by protecting against oxidative stress in colitis. Redox Biology 37 (2020): 101760.

10. Moret-Tatay I, Ibora M, Cerrillo E, Tortosa L, Ns P, Beltrán B. Possible biomarkers in blood for Crohn's disease: Oxidative stress and microRNAs-Current evidences and further aspects to unravel. Oxidative medicine and cellular longevity 2016 (2016).

11. Kruidenier L, Kuiper I, van Duijn W, et al. Differential mucosal expression of three superoxide dismutase isoforms in inflammatory bowel disease. J Pathol 2003; 201: 7-16.

12. Mulder TP, Verspaget HW, Janssens AR, de Bruin PA, Peña AS, Lamers CB. Decrease in two intestinal copper/zinc containing proteins with antioxidant function in inflammatory bowel disease. Gut 1991; 32(10): 1146-50.

13. Kruidenier L, Kuiper I, Lamers CB, Verspaget HW. Intestinal oxidative damage in inflammatory bowel disease: semiquantification, localization, and association with mucosal antioxidants. J Pathol 2003 201: 28-36.

14. Iantomasi T, Marraccini P, Favilli F, Vincenzini MT, Ferretti P, Tonelli F. Glutathione metabolism in Crohn's Disease. Biochemical Medicine and Metabolic Biology 1994; 53(2): 87-91.
15. Iborra M, Moret I, Rausell F, et al. Role of oxidative stress and antioxidant enzymes in Crohn's disease. Biochemical Society Transactions 2011; 39(4): 1102-6.

16. Godet PG, May GR, Sutherland LR. Meta-analysis of the role of oral contraceptive agents in inflammatory bowel disease. Gut 1995; 37(5): 668-73.

17. Khalili H, Higuchi LM, Ananthakrishnan AN, Richter JM, et al. Oral contraceptives, reproductive factors and risk of inflammatory bowel disease. Gut 2013; 62(8): 1153-9.

18. Unfer TC, Figueiredo CG, Zanchi MM, et al. Estrogen plus progestin increase superoxide dismutase and total antioxidant capacity in postmenopausal women. Climacteric 2015; 18(3):379-88.

19. Strehlow K, Rotter S, Wassmann S, et al. Modulation of antioxidant enzyme expression and function by estrogen. Circulation Research 2003; 93(2): 170-7.

20. Underner M, Perriot J, Cosnes J, Beau P, Peiffer G, Meurice J-C. Smoking, smoking cessation and Crohn's disease. Presse Med 2016; 45(4 Pt 1): 390-402.

21. Kuenzig ME, Lee SM, Eksteen B, Seow CH, et al. Smoking influences the need for surgery in patients with the inflammatory bowel diseases: a systematic review and meta-analysis incorporating disease duration. BMC Gastroenterol 2016; 16(1): 143.

22. Lakatos PL, Szamosi T, Lakatos L. Smoking in inflammatory bowel diseases: good, bad or ugly? World J Gastroenterol 2007; 13(46): $6134-9$.

23. Yamamoto, T, Keighley MR. Smoking and disease recurrence after operation for Crohn's disease. British Journal of Surgery 2000; 87(4): 398-404.

24. Bureš J, Fixa B, Komárková O, Fingerland A. Non-smoking: a feature of ulcerative colitis. Br Med J 1982; 285(6339): 440.

25. Lin Y, Liu X, Cheng Y, Yang J, Huo Y, Zhang C. Involvement of MicroRNAs in hydrogen peroxide-mediated gene regulation and cellular injury response in vascular smooth muscle cells. The Journal of Biological Chemistry 2009; 284(12): 7903-13.

26. Wang L, Huang $H$, Fan $Y$, et al. Effects of downregulation of microRNA-181a on $\mathrm{H}_{2} \mathrm{O}_{2}$-induced $\mathrm{H} 9 \mathrm{c} 2$ cell apoptosis via the mitochondrial apoptotic pathway. Oxid Med Cell Longev 2014; 2014: 960362.

27. Christian P, Su O. MicroRNA regulation of mitochondrial and ER stress signaling pathways: implications for lipoprotein metabolism in metabolic syndrome. American Journal of Physiology - Endocrinology and Metabolism 2014; 307(9): 729-37. 